\title{
A carboxymethyl dextran-based polymeric conjugate as the antigen carrier for cancer immunotherapy
}

Jung Min Shin ${ }^{1 \dagger}$, Seok Ho Song ${ }^{1 \dagger}$, N. Vijayakameswara Rao ${ }^{1}$, Eun Sook Lee ${ }^{2}$, Hyewon Ko ${ }^{2}$ and Jae Hyung Park ${ }^{1,2,3^{*}}$

\begin{abstract}
Background: Antigen-specific cytotoxic T lymphocytes (CTLs), which eliminate target cells bearing antigenic peptides presented by surface major histocompatibility complex $(\mathrm{MHC})$ class I molecules, play a key role in cancer immunotherapy. However, the majority of tumors are not immunologically rejected since they express self-antigens which are not recognized by CTLS as foreign. To foreignize these tumors for CTL-mediated immunological rejection, it is essential to develop carriers that can effectively deliver foreign antigens to cancer cells.
\end{abstract}

Methods: A polymeric conjugate, composed of a carboxymethyl dextran (CMD) as the backbone and ovalbumin (OVA) as a model foreign antigen, was prepared to investigate its potential as the antigen carrier for cancer immunotherapy.

Results: An in vitro cellular uptake study showed that the conjugate was successfully taken up by TC-1 cervical cancer cells. When CMD-OVA was systemically administered to tumor-bearing mice, the strong fluorescence signal was observed at the tumor site over the whole period of time period, suggesting high tumor targetability of the conjugate. Compared to free OVA, CMD-OVA induced significantly higher antigen presentation at the tumor site.

Conclusions: The CMD-OVA conjugate can effectively deliver the antigen to the tumor site, implying its high potential as the antigen carrier for cancer immunotherapy.

Keywords: Carboxymethyl dextran, Cancer immunotherapy, Antigen delivery

\section{Background}

In recent years, cytotoxic $\mathrm{T}$ lymphocytes (CTL) have been extensively studied for their ability to destroy target cells bearing antigenic peptides presented by surface major histocompatibility complex (MHC) class I molecules [1-3]. Taking advantage of the unique functions of CTLs, chimeric antigen receptor (CAR)-T and adoptive cell therapy (ACT) have been used in clinical trials [4-6]. Although they have drawbacks such as high cost and limited use in autologous therapy, these therapeutic approaches are useful for cancer treatment.

Unfortunately, tumors can avoid CTL recognition because they have self-antigen on their surfaces. For

\footnotetext{
* Correspondence: jhpark1@skku.edu

†ung Min Shin and Seok Ho Song contributed equally to this work.

'School of Chemical Engineering, Sungkyunkwan University, Suwon 16419, Republic of Korea

${ }^{2}$ Department of Health Science and Technology, SAIHST, Sungkyunkwan

University, Suwon 16419, Republic of Korea

Full list of author information is available at the end of the article
}

CTL-mediated immunological rejection of tumors by foreignization, it is essential to develop carriers that can deliver foreign antigens to the cancer cells $[7,8]$. To date, no significant effort has been devoted to the development of a tumor-specific intracellular delivery system for these antigens.

Polymeric conjugates with targeting ligands have been studied as drug carriers for cancer therapy. They accumulate passively at the tumor site via enhanced permeation and retention effect and undergo uptake via receptor-mediated endocytosis by tumor cells [9-14]. Among polymeric materials, considerable effort has focused on using carboxymethyl dextran (CMD) as a drug carrier for cancer therapy and imaging because of its high biocompatibility, excellent water solubility, and biodegradability [15-17]. In particular, owing to its multiple functional groups allowing for facile chemical modification, CMD has been

(c) The Author(s). 2018 Open Access This article is distributed under the terms of the Creative Commons Attribution 4.0 International License (http://creativecommons.org/licenses/by/4.0/), which permits unrestricted use, distribution, and 
extensively used to develop polymeric conjugates as the nanomedicines [18, 19].

Herein, in an attempt to foreignize the cancer cells, we prepared a CMD-based polymeric conjugate with ovalbumin (OVA) as a model foreign antigen (Fig. 1). Its uptake behavior by cancer cells have been assessed using confocal microscopy. After systemic administration of the conjugate into the tumor-bearing mice, it's in vivo biodistribution was observed using the optical imaging technique. Also, in vivo antigen presentation was observed to estimate the potential of the conjugate as the antigen carrier for cancer immunotherapy.

\section{Methods}

\section{Materials}

CMD sodium salt $\left(M_{n}=10,000-20,000 \mathrm{Da}\right)$, OVA, 1-eth yl-3-(3-dimethylaminopropyl)carbodiimide-hydrochloride (EDC. $\mathrm{HCl}), N$-hydroxysuccinimide (NHS), sodium cyanoborohydride $\left(\mathrm{NaBH}_{3} \mathrm{CN}\right)$, and fluorescein isothiocyanate (FITC) were purchased from Sigma-Aldrich (St. Louis, MO, USA). The near-infrared fluorescent (NIRF) probe, cyanine 5.5 (Cy5.5), was purchased from Amersham Bioscience (Piscataway, NJ, USA). The water used in this study was prepared by an AquaMax-Ultra Water Purification System (Anyang, Republic of Korea). All

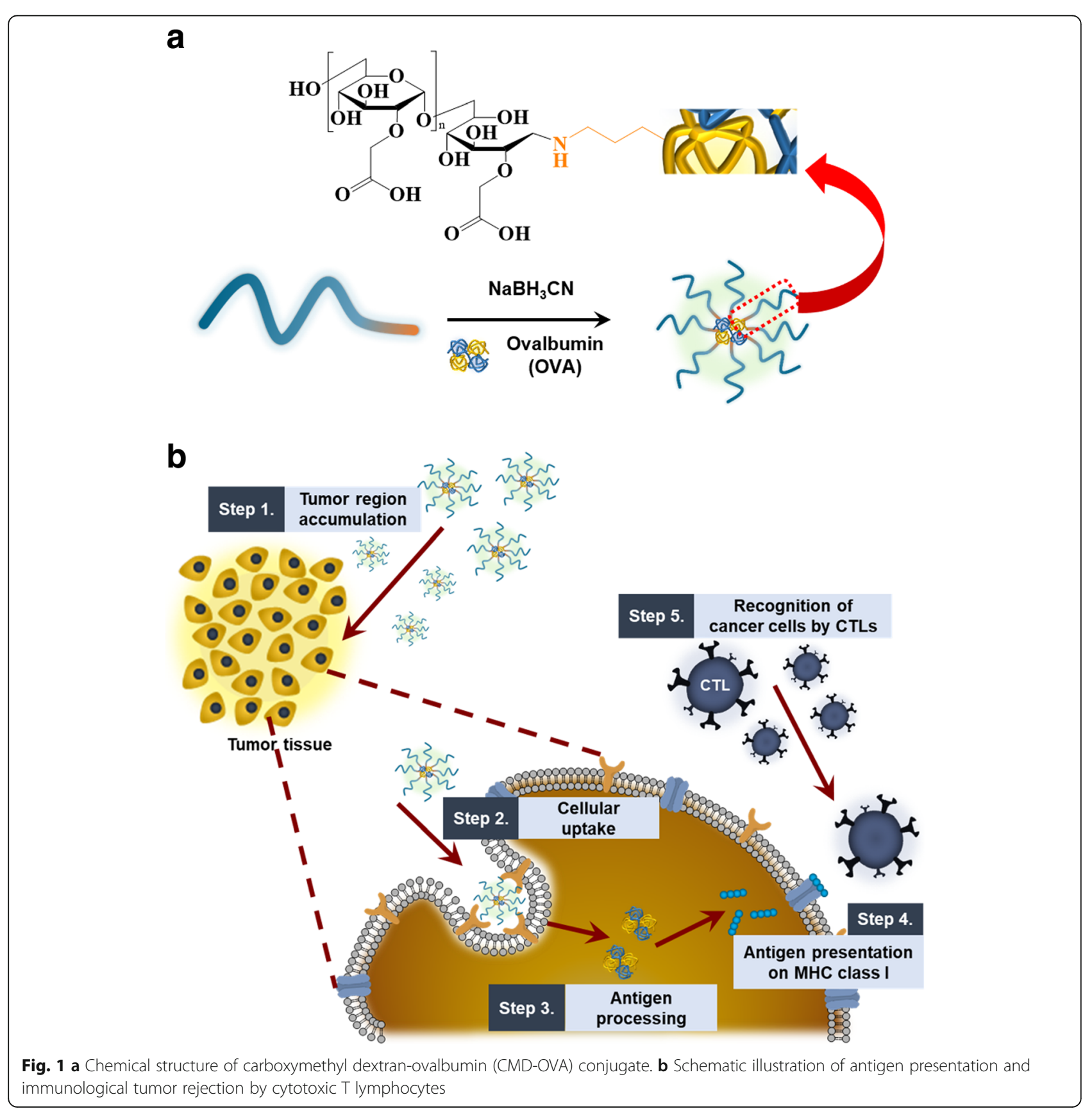


other chemicals were prepared by commercial sources, and they were used as received without purification.

\section{Preparation of CMD-OVA conjugate}

OVA was chemically affixed to CMD via reductive amination between the reducing end group of $\mathrm{CMD}$ and the amino group of OVA, in the presence of $\mathrm{NaBH}_{3} \mathrm{CN}$ as a reducing agent. Briefly, the CMD (82.65 mg) solution in $40 \mathrm{ml}$ of borate buffer $(\mathrm{pH} 8.5,0.4 \mathrm{M} \mathrm{NaCl})$ was mixed with OVA (25 mg), dissolved in $1 \mathrm{~mL}$ of borate buffer ( $\mathrm{pH}$ 8.5). Sodium cyanoborohydride $(100 \mathrm{mg}$ ) was added to the reaction mixture, which was then allowed to stir for 5 days at $40{ }^{\circ} \mathrm{C}$. The conjugate was obtained by dialysis using deionized water for 3 days in cellulose membrane bag (molecular weight cut off = $50 \mathrm{kDa}$ ), followed by lyophilization. Prior to use, the conjugate was stored at $-20{ }^{\circ} \mathrm{C}$.

\section{Cellular uptake behavior of CMD-OVA conjugate}

To investigate the internalization of the CMD-OVA conjugate in vitro, the conjugate was labeled with FITC. TC-1 cells (a cervical cancer cell line expressing the E7 protein of human papilloma virus type 16) were cultured in RPMI 1640 medium containing $10 \%(\mathrm{v} / \mathrm{v})$ fetal bovine serum (FBS) and 1\% (v/v) penicillin and streptomycin solution in a humidified cell culture incubator at $37{ }^{\circ} \mathrm{C}$. The cells, seeded at $2 \times$ $10^{5}$ cells/well in 6-well plates for 1 day, were treated either with FITC-labeled OVA or CMD-OVA in RPMI 1640 media without FBS for $3 \mathrm{~h}$. The cells were then washed twice using PBS containing $\mathrm{Ca}^{2+}$ and $\mathrm{Mg}^{2+}$, and fixed using a $4 \%$ paraformaldehyde solution. The cellular uptake of the CMD-OVA conjugate was visualized using confocal laser scanning microscopy (CLSM) (TCS SP8 HyVolution, Leica Microsystems, Wetzlar, Germany) and quantified using flow cytometry (Guava easyCyte, EMD Millipore, Billerica, MA, USA).

\section{In vivo biodistribution}

The conjugate was labeled with Cy5.5 as follows. In brief, the hydrazide derivative of $\mathrm{Cy} 5.5$ was reacted with carboxylic groups of CMD in the presence of EDC overnight in the dark. The reaction mixtures were then dialyzed (molecular weight cut off $=3 \mathrm{kDa}$ ) against deionized water to remove non-reacted Cy5.5 and EDC. The purified conjugate was stored at $-20{ }^{\circ} \mathrm{C}$, prior to use. To observe the in vivo tumor-homing ability of the conjugate, a TC-1 flank tumor model was prepared by the subcutaneous inoculation of a cell suspension $\left(1 \times 10^{6}\right.$ cells for each mouse) in RPMI medium $(100 \mu \mathrm{L})$ into athymic nude mice (6-week-old, female). When the tumor volumes reached $150-200 \mathrm{~mm}^{3}, 200 \mu \mathrm{L}$ of physiological saline containing Cy5.5-labeled polymeric conjugate was injected into the tail vein of each mouse. The conjugate was visualized at predetermined time points by scanning the mice using a Lago X system (Spectral Instruments Imaging, Tucson, AZ, USA) with a $670 \mathrm{~nm}$ LED. The tumors and major organs (liver, heart, lung, spleen, and kidney) were excised from the mice at $48 \mathrm{~h}$ post-injection to quantitatively assess the ex vivo tissue distribution of the conjugate by measuring the average fluorescence intensity over the region of interest.

\section{In vivo antigen presentation}

To prepare the tumor-bearing animal model, TC-1 cells $\left(2 \times 10^{5}\right.$ cells for each mouse) were subcutaneously injected into C57BL/6 mice (8-week-old, female). After 10 days, each conjugate (OVA or CMD-OVA) was dissolved in saline containing $100 \mu \mathrm{g}$ of OVA and was then systemically administered through the tail vein. At $24 \mathrm{~h}$ post-injection, the tumor tissue was removed, dissociated by a cell strainer, and washed twice with PBS. The

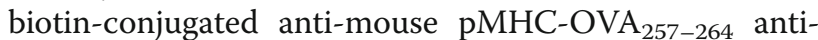
body was used to stain $\mathrm{D}^{\mathrm{b}} / \mathrm{OVA}_{257-264}$ complexes. For quantification of the in vivo OVA presentation, flow cytometry analysis was performed.

\section{Statistical analysis}

The statistical significance of experimental results was assessed using one-way analysis of variance (ANOVA), and a $p$ value $<0.05$ was regarded as significant (indicated with an asterisk (*) in the corresponding figures).

\section{Results}

\section{Preparation and characterization of the CMD-OVA conjugate}

The CMD-OVA conjugate was prepared as a carrier that can effectively deliver antigens to cancer cells for CTL-mediated immunological rejection by foreignization. Of the various biocompatible polymers, CMD was chosen as the backbone due to its unique structure, high biocompatibility, excellent water solubility, and biodegradability. OVA was used as a model antigen, chemically attached to the reducing end group of the CMD chain via reductive amination in the presence of $\mathrm{NaBH}_{3} \mathrm{CN}$. The OVA content of CMD-OVA was directly quantified by the bicinchoninic acid assay as $133 \mu \mathrm{g}$ of OVA per $1 \mathrm{mg}$ of CMD-OVA.

\section{In vitro cellular uptake}

For CTL-mediated cell death, foreign antigens should be processed inside the target cells, followed by presentation on their surfaces as part of the MHC class I complex. Therefore, if the target cells do not internalize the conjugate, CTLs cannot identify and destroy them. To explore the cellular uptake of the conjugate in vitro, CMD-OVA was incubated with TC-1 cells, and its uptake was observed utilizing CLSM (Fig. 2a). CMD-OVA 


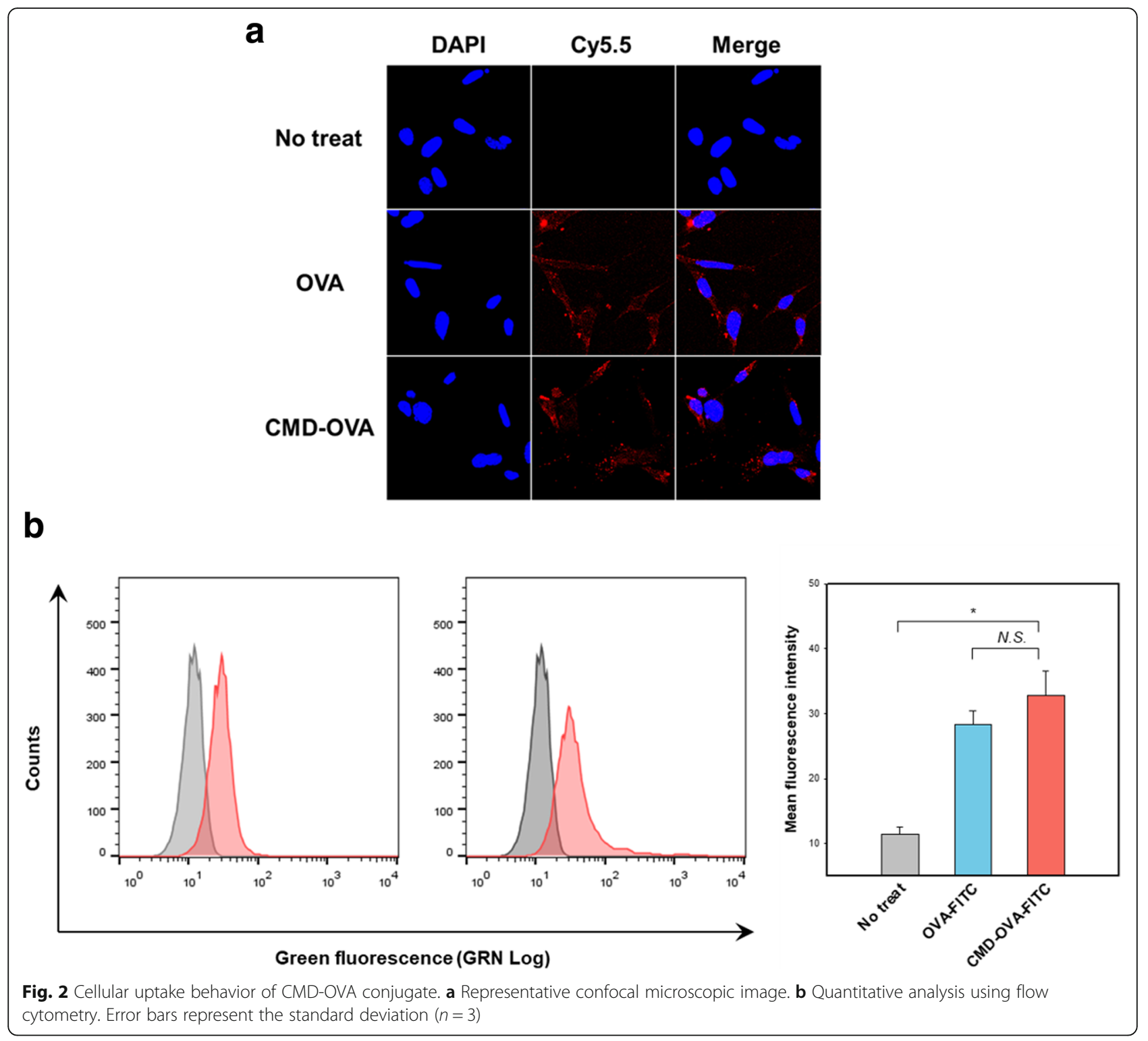

showed a similar intracellular fluorescence signal to the OVA-FITC control. The cellular uptake of the conjugate was also measured using flow cytometry (Fig. 2b). Quantitative analysis indicated that the amount of the conjugate, taken up by the cancer cells, were comparable to that of OVA.

\section{In vivo biodistribution of the CMD-OVA conjugate}

To examine the in vivo biodistribution, Cy5.5-labeled CMD-OVA or OVA was systemically injected into the TC-1 tumor-bearing mice. The fluorescence images of the tumor site were acquired by using a non-invasive optical imaging system. As shown in Fig. 3a, compared to OVA, the CMD-OVA conjugate exhibited stronger fluorescence signals in the entire bodies of the mice for the whole test period, implying prolonged circulation of CMD-OVA. It is worthy to note that strong fluorescence signals of CMD-OVA were observed at the tumor site (the white dotted line), whereas no significant signals were found for OVA. As demonstrated by ex vivo images of the major organs, most of OVA was accumulated in liver, where minimal amount of CMD-OVA was detected (Fig. 3b). This result implies that the CMD-OVA conjugate can effectively accumulate at tumor sites, thus possessing potential to effectively deliver the antigen to the tumor.

In vivo antigen presentation of CMD-OVA conjugate The OVA antigen presentation of TC-1 cells, treated with CMD-OVA or OVA, was measured by flow cytometry. After cell isolation from mice, TC-1 cells were stained with an anti-mouse MHC-OVA peptide (pMHC-OVA $257-264$ ) antibody, which specifically binds to 


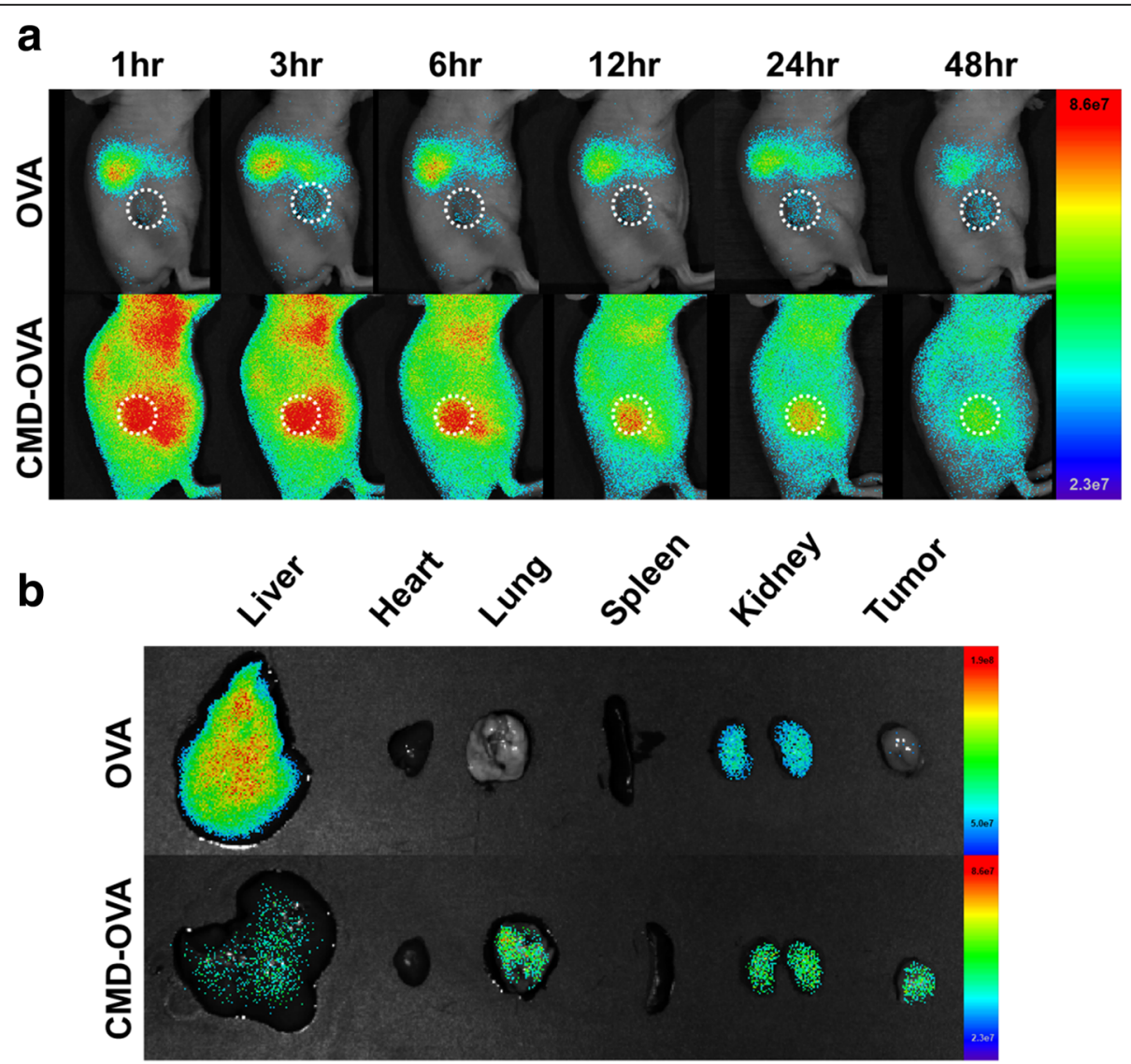

Fig. 3 In vivo distribution of CMD-OVA conjugate. a Whole body near-infrared fluorescence (NIRF) image of CMD-OVA conjugate as a function of time. The white dotted line indicated tumor site. $\mathbf{b}$ Ex vivo NIRF images of tumor and major organs after $48 \mathrm{~h}$

mouse MHC class I-OVA peptide $\left(\mathrm{OVA}_{257-264}\right)$ complexes. Relative OVA antigen presentation was quantified using flow cytometry (Fig. 4). The CMD-OVA conjugate induced much higher $\mathrm{OVA}_{257-264}$ presentation, compared with free OVA. Overall, these data suggest that the CMD-OVA conjugate is efficiently taken up by tumor cells, followed by the enhanced presentation of a passenger antigen (OVA)-MHC class I complex.

\section{Discussion}

In recent years, owing to their unique characteristics, polymeric conjugates have gained attention for biomedical applications such as drug delivery and tissue engineering. Particularly, several conjugates have been approved by FDA because chemical attachment of biocompatible polymers to bioactive agents have been demonstrated to prolong their blood circulation, resulting in enhanced biological half-lives [20, 21].

In order to overcome current limitations of conventional chemotherapy, immunotherapeutic approaches have recently emerged based on immune checkpoint inhibitor, CAR $\mathrm{T}$ cell, and neoantigen [22-24]. For example, neoantigens derived from cancer cells have received attention because they are easily distinguished from self-antigens, leading to elimination of cancer cells by CTLs. In this study, the biocompatible CMD-based antigen carrier has been prepared to investigate its potential for cancer immunotherapy. From the in vitro cellular uptake study, it was found that the conjugate was effectively taken up by the cancer cells, implying that the conjugate can deliver the antigen into the intracellular compartments for antigen processing. After systemic administration into the tumor-bearing mice, the conjugate was efficiently accumulated at the tumor site, compared to free OVA. This high tumor targetability of the conjugate might account for significant antigen presentation in vivo (Fig. 4), which may facilitate recognition of the cancer cells by CTLs.

This foreign antigen delivery technology can be applied to various diseases. For example, for rheumatoid arthritis, activated macrophages are primarily responsible for inflammatory responses. If foreign antigens can be delivered to the activated macrophages, it may cause their apoptotic cell death by 


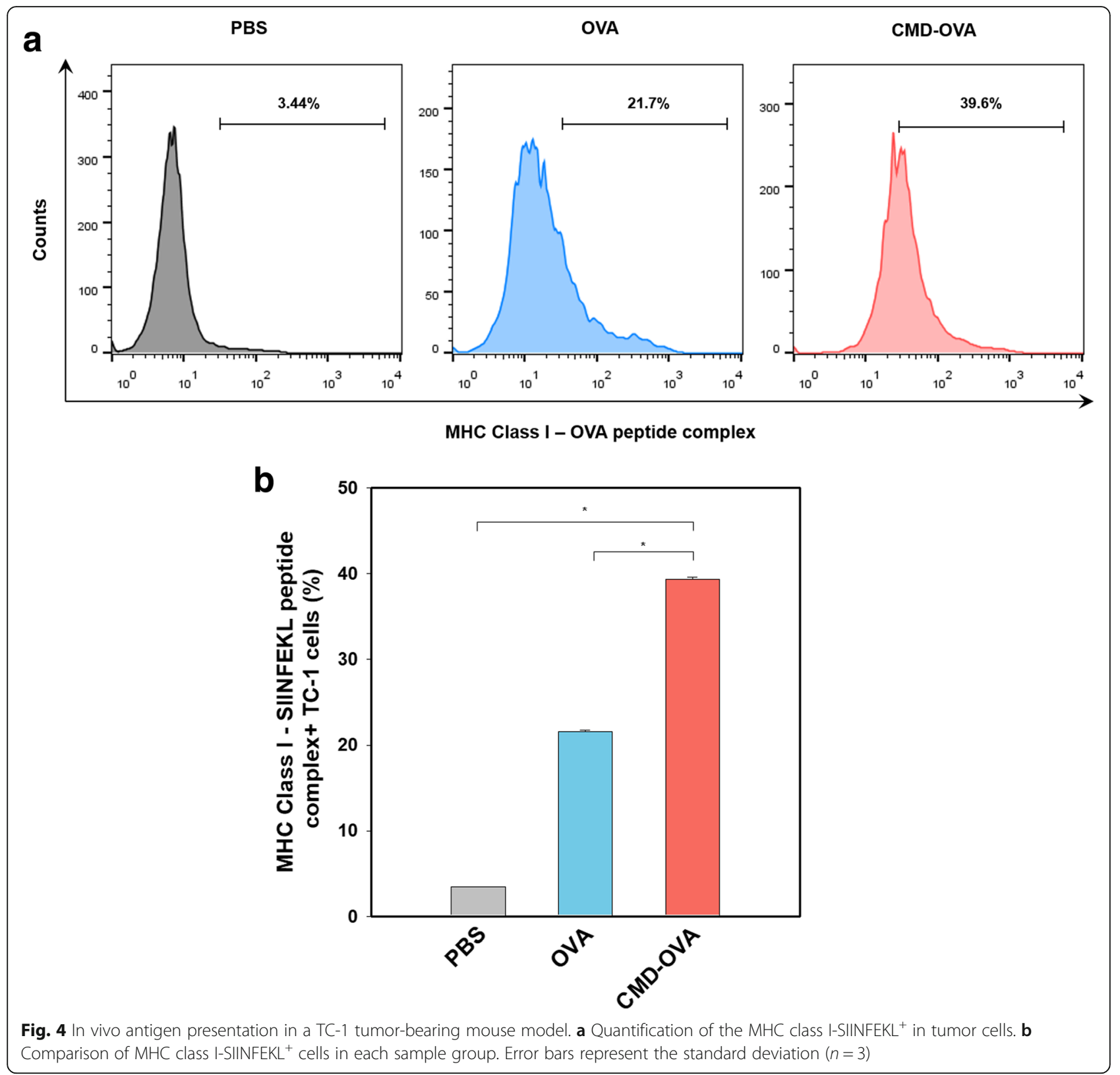

CTL-mediated immune responses, resulting in suppression of the symptoms by rheumatoid arthritis. Overall, this foreign antigen delivery technology based on the polymeric conjugates would be highly useful for treatments of various intractable diseases.

\section{Conclusion}

The CMD-OVA conjugate was successfully synthesized by the reductive amination reaction. The conjugate was efficiently taken up by tumor cells, followed by the enhanced presentation of a model foreign antigen (OVA) as part of the MHC class I complex. Our findings based on a CMD-OVA polymeric conjugate as a foreign antigen-delivery system could serve as a platform technology for treatments of cancer.

\section{Abbreviations}

CLSM: Confocal laser scanning microscopy; CMD: Carboxymethyl dextran; CTLs: Cytotoxic T lymphocytes; Cy5.5: Cyanine 5.5; EDC.HCl: 1-ethyl-3-(3dimethylaminopropyl)carbodiimide.hydrochloride; FBS: Fetal bovine serum; FITC: Fluorescein isothiocyanate; MHC: Major histocompatibility complex: NaBH3CN: Sodium cyanoborohydride; NHS: N-hydroxysuccinimide; NIRF: Near-infrared fluorescent; OVA: Ovalbumin

\section{Funding}

This research was supported by the Basic Science Research Programs of the National Research Foundation of Korea (NRF), Republic of Korea (20100027955 and 2018R1A2B3006080). 


\section{Availability of data and materials}

The datasets used and/or analyzed during the current study are available from the corresponding author on reasonable request.

\section{Authors' contributions}

JMS and SHS coordinated the research. NVR and ESL helped in writing manuscript. HK helped animal experiment. All of this work is supervised by Professor JHP. All authors read and approved the final manuscript.

\section{Ethic approval and consent to participate}

Not applicable

\section{Consent for publication}

Not applicable

\section{Competing interests}

The authors declare that they have no competing interests.

\section{Publisher's Note}

Springer Nature remains neutral with regard to jurisdictional claims in published maps and institutional affiliations.

\section{Author details}

'School of Chemical Engineering, Sungkyunkwan University, Suwon 16419, Republic of Korea. ${ }^{2}$ Department of Health Science and Technology, SAIHST, Sungkyunkwan University, Suwon 16419, Republic of Korea. ${ }^{3}$ School of Chemical Engineering, Sungkyunkwan University, Suwon 440-746, Republic of Korea.

Received: 23 May 2018 Accepted: 30 July 2018

Published online: 14 August 2018

\section{References}

1. Friedl $P$, den Boer AT, Gunzer M. Tuning immune responses: diversity and adaptation of the immunological synapse. Nat Rev Immunol. 2005;5:532.

2. Kang $\mathrm{TH}$, Lee $\mathrm{JH}$, Song $\mathrm{CK}$, Han HD, Shin BC, Pai SI, Hung C-F, Trimble C, Lim J-S, Kim TW, Wu TC. Epigallocatechin-3-Gallate enhances CD8(+) T cellmediated antitumor immunity induced by DNA vaccination. Cancer Res. 2007:67:802-11.

3. Jondal M, Schirmbeck R, Reimann J. MHC class I-restricted CTL responses to exogenous antigens. Immunity. 1996;5:295-302.

4. Brudno JN, Kochenderfer JN. Chimeric antigen receptor T-cell therapies for lymphoma. Nat Rev Clin Oncol. 2017:15:31.

5. Neelapu SS, Tummala S, Kebriaei P, Wierda W, Gutierrez C, Locke FL, Komanduri KV, Lin Y, Jain N, Daver N, Westin J, Gulbis AM, Loghin ME, de Groot JF, Adkins S, Davis SE, Rezvani K, Hwu P, Shpall EJ. Chimeric antigen receptor T-cell therapy — assessment and management of toxicities. Nat Rev Clin Oncol. 2017;15:47

6. Rosenberg SA, Restifo NP. Adoptive cell transfer as personalized immunotherapy for human cancer. Science. 2015;348:62-8.

7. Lee Y-H, Yoon HY, Shin JM, Saravanakumar G, Noh KH, Song K-H, Jeon J-H, Kim D-W, Lee K-M, Kim K, Kwon IC, Park JH, Kim TW. A polymeric conjugate foreignizing tumor cells for targeted immunotherapy in vivo. J Control Release. 2015:199:98-105.

8. Shin JM, Oh SJ, Kwon S, Deepagan VG, Lee M, Song SH, Lee H-J, Kim S, Song K-H, Kim TW, Park JH. A PEGylated hyaluronic acid conjugate for targeted cancer immunotherapy. J Control Release. 2017;267:181-90.

9. Bourzac K. Nanotechnology: carrying drugs. Nature. 2012;491:S58.

10. Maeda H, Wu J, Sawa T, Matsumura Y, Hori K. Tumor vascular permeability and the EPR effect in macromolecular therapeutics: a review. J Control Release. 2000;65:271-84.

11. Rao N V, Dinda H, Ganivada MN, Das Sarma J, Shunmugam R. Efficient approach to prepare multiple chemotherapeutic agent conjugated nanocarrier. Chemical Communications. 50((2014)):13540-3.

12. Rao N V, Ganivada MN, Sarkar S, Dinda H, Chatterjee K, Dalui T, Das Sarma J, Shunmugam R. Magnetic Norbornene Polymer as Multiresponsive Nanocarrier for Site Specific Cancer Therapy. Bioconjug Chem. 25((2014)):276-85.

13. Shin JM, Hwang SR, Heo R, Saravanakumar G, Park JH. Amphiphilic hyaluronic acid derivative with the bioreducible bond: synthesis and its implication for intracellular drug delivery. Polym Degrad Stab. 2014;109:398-404.
14. J.M. Shin, S.H. Kim, T. Thambi, D.G. You, J. Jeon, J.O. Lee, B.Y. Chung, D.G. Jo, J.H. Park, A hyaluronic acid-methotrexate conjugate for targeted therapy of rheumatoid arthritis, Chemical communications (Cambridge, England), 50 (2014) 7632-7635.

15. Thambi T, You DG, Han HS, Deepagan VG, Jeon SM, Suh YD, Choi KY, Kim K, Kwon IC, Yi GR, Lee JY, Lee DS, Park JH. Bioreducible Carboxymethyl dextran nanoparticles for tumor-targeted drug delivery. Advanced Healthcare Materials. 2014;3:1829-38.

16. Wang Z-H, Zhu Y, Chai M-Y, Yang W-T, Xu F-J. Biocleavable comb-shaped gene carriers from dextran backbones with bioreducible ATRP initiation sites. Biomaterials. 2012;33:1873-83.

17. Son S, Rao NV, Ko H, Shin S, Jeon J, Han HS, Nguyen VQ, Thambi T, Suh YD, Park JH. Carboxymethyl dextran-based hypoxia-responsive nanoparticles for doxorubicin delivery. Int J Biol Macromol. 2018;110:399-405.

18. Okuno S, Harada M, Yano T, Yano S, Kiuchi S, Tsuda N, Sakamura Y, Imai J, Kawaguchi T, Tsujihara K. Complete regression of Xenografted human carcinomas by Camptothecin analogue-Carboxymethyl dextran conjugate (T-0128). Cancer Res. 2000;60:2988-95.

19. Gaowa A, Horibe T, Kohno M, Tabata Y, Harada H, Hiraoka M, Kawakami K. Enhancement of anti-tumor activity of hybrid peptide in conjugation with carboxymethyl dextran via disulfide linkers. Eur J Pharm Biopharm. 2015:92:228-36

20. Alconcel SN, Baas AS, Maynard HD. FDA-approved poly (ethylene glycol)protein conjugate drugs. Polym Chem. 2011:2:1442-8.

21. Bobo D, Robinson KJ, Islam J, Thurecht KJ, Corrie SR. Nanoparticle-based medicines: a review of FDA-approved materials and clinical trials to date. Pharm Res. 2016;33:2373-87

22. Sondak VK, Smalley KS, Kudchadkar R, Grippon S, Kirkpatrick P. Ipilimumab: Nature Publishing Group; 2011

23. Davila ML, Riviere I, Wang X, Bartido S, Park J, Curran K, Chung SS, Stefanski J, Borquez-Ojeda O, Olszewska M. Efficacy and toxicity management of 19$28 z$ CAR T cell therapy in B cell acute lymphoblastic leukemia. Sci Transl Med. 2014:6(224):224ra225.

24. Ott PA, Hu Z, Keskin DB, Shukla SA, Sun J, Bozym DJ, Zhang W, Luoma A, Giobbie-Hurder A, Peter L. An immunogenic personal neoantigen vaccine for patients with melanoma. Nature. 2017;547:217.

\section{Ready to submit your research? Choose BMC and benefit from:}

- fast, convenient online submission

- thorough peer review by experienced researchers in your field

- rapid publication on acceptance

- support for research data, including large and complex data types

- gold Open Access which fosters wider collaboration and increased citations

- maximum visibility for your research: over $100 \mathrm{M}$ website views per year

At $\mathrm{BMC}$, research is always in progress.

Learn more biomedcentral.com/submissions 